\title{
An analysis of the accelerations of a projectile in a gun tube by direct measurements and telemetry of the data
}

\author{
Mathilda Liennard ${ }^{1}$, Ophélie Chevalier ${ }^{2}$, André Langlet ${ }^{3,}{ }^{*}$, Yann Guilmard $^{2}$, and Marcel Mansion ${ }^{4}$ \\ ${ }^{1}$ DGA-Techniques Terrestres, Echangeur de Guerry, 18000 Bourges, France \\ 2 Nexter-Systems, 7 route de Guerry, 18023 Bourges Cedex, France \\ ${ }^{3}$ LaMé EA-7494 Université d'Orléans, Université de Tours, INSA CVL, 63 Avenue de Lattre de Tassigny, 18000 Bourges, \\ France \\ 4 ATCOM-Télémétrie, 15 rue Jean Bertin, 45430 Chécy, France
}

Received: 19 April 2017 / Accepted: 9 March 2018

\begin{abstract}
In this study, transverse and longitudinal accelerations of a full caliber-shell shot by a mediumcaliber gun were measured directly during the internal ballistic (IB) phase. The data were transmitted outside the tube by modulation of LASER light emission and recoded into acceleration values. The results were analyzed through the numerical simulation of the response of the tube traversed by the projectile and with the internalballistic data providing the longitudinal projectile acceleration. The analysis reveals that the in-bore projectile movements are both influenced by the tube geometry (curvature and straightness defects) and by the tube response during the internal ballistic phase. However, in this work, the tube response appeared to be of greater influence on the projectile dynamics than the tube initial static geometry. Results provide basis for further calibration studies.
\end{abstract}

Keywords: Experiments / gun dynamics / projectile / telemetry / internal ballistic / finite element simulations

\section{Introduction}

The main components of a gun-weapon system are the gun barrel and the ammunition. When the projectile (shell) moves in the barrel [internal ballistic (IB) phase], the response of the system is characterized by its short duration and by the very large accelerations and pressure, which reach about $8 \times 10^{5} \mathrm{~m} / \mathrm{s}^{2}$ and $900 \mathrm{MPa}$ within a maximum duration of $5 \mathrm{~ms}$ for the $25 \mathrm{~mm}$ shells studied in this work ${ }^{1}$. During the first milliseconds of a shot, the propulsive gas and the moving shell, both apply highly dynamic actions on the gun tube. In reaction, the barrel applies complex loads on the moving projectile. Resulting from this coupling, the IB phase can be different from the ideal one, which is expected for the maximum efficiency of the shot in order to hit the target. In effect, the exit conditions of the projectile may be largely altered and far from those insuring optimal accuracy of the weapon system.

\footnotetext{
* Corresponding author: e-mail:

andre. langlet@univ-orleans.fr

${ }^{1}$ Due to industrial property concerns and for reasons of confidentiality, some numerical data or results are not communicated explicitly.
}

Accuracy is influenced by the displacement, velocity, acceleration, and rotation (due to the rifling) vectors of the projectile when it exits the gun tube. These vectors are the initial conditions for the trajectory towards the target.

The functioning of a weapon system is influenced by numerous factors, which include at first the IB parameters (driving pressure, acceleration, velocity, spin rate, projectile design, etc.) and the tube structural response. By coupling, the projectile movements are influenced by those of the tube. Meanwhile, transverse motions of the projectile in the gun tube differing from the movement of it may occur. In-bore balloting can be caused by any one or more of the following conditions: projectile center of gravity and principal axis offset from the bore centerline; projectiles, as manufactured, are not perfectly concentric; projectile/gun tube clearances may exist at projectile surfaces; insufficient stiffness of the projectile/gun tube interface will exacerbate conditions caused by minimal balloting; projectile wear produces or widens gaps (clearances) between the projectile bourrelet surfaces and the gun bore; gun tube curvature exists in all gun tubes [1,2]); gas dynamics produce irregular pressures at the projectile base [3].

To define optimizing actions (such as corrective actions on the fire-control system), ideally, the transient displacements of the tube and of the projectile should be known at every time of the IB phase. 
Since guns are slender bodies exhibiting flexural responses and radial expansion, a lot of studies based on the theory of beams (for example, [4], [5], [6]) or shells ([7], [8]) interacting with a moving mass or moving oscillators representing the projectile, have been published. Although these studies based on specialized theories, can provide valuable qualitative insights on the coupled tube-projectile response, these approaches failed to capture the complexity of the 3D response. On this subject, few studies are published in the open literature, providing few data for the analysis of real firing system. The behavior of Armor Piercing Fin Discarding Sabot projectile (APFSDS) seems to be more studied than that of full caliber shells. The reason is that the oscillations of the APFSDS projectile are more important due to the slipping of the sabots on the tube wall and to the slenderness of the arrow projectile [9].

Both the modeling and the experimental approaches face the difficulty of determining the translational and rotational degree of freedom along direction normal to the longitudinal axis. Although the formulation of these displacements can be found in previous works [10] a reliable solution for specific gun type remain to be obtained. Measuring strains on the external wall of the barrel, or measuring the muzzle displacement are quite easy tasks. It has been proved that these measurements are reasonably correlated with finite element model (FEM) simulations, for example $[11,12]$, or mixed lumped/FEM models (for example, developed with SIMBAD [13]). The FEM models [11] and [12] used explicit solvers based on the Ls-Dyna commercial code with hexahedral elements and elastoplastic material behavior. Results of such simulations enable to forecast lifetime of gun barrel limited by damage accumulation due to the major stresses identified in the barrel [11] or wear of the barrel [12]. Assessing the transverse projectile kinematic remains a challenging problem which is not completely solved. In effect the deviations of the real in-bore projectile trajectory from the ideal one remains to be determined to optimize the efficiency of all weapon systems.

Several experimental methods may be used for assessing the projectile displacements in the IB phase.

For example, hyperfrequency interferometry associated with Doppler Radars or X-ray imaging (through the tube wall) are proposed by [14] and [15], respectively. The main disadvantage of these methods is that only the longitudinal displacement can be obtained. Another solution used with $105 \mathrm{~mm}$ caliber shells is to be placed on a reflecting surface on the projectile nose and light it with a LASER beam. The reflected LASER is deviated by a mirror and then recorded [16]. With the same idea [17], fitted a LASER transmitter in the projectile nose and recorded the plane beam trajectory intercepted by a mirror. The analysis of the plane trajectory of the beam on the mirror enables to infer the nose projectile movement. However, these solutions are dependent on the accuracy of the recorded LASER spot, the size of which may be of the same order of the investigated displacements (or even larger). Results of angular acceleration measurements have been presented by [18] for field artillery guns. In principle, analysis of the angular accelerations may lead to the translational displacements, provided a part of the longitudinal kinematic is known in advance by mean of pre-determined time function [19]. However, the permissible angular accelerations supported by the accelerometers are less than the maximum accelerations applied in medium caliber gun (from 20 to $40 \mathrm{~mm}$ caliber) which reach about $750000 \mathrm{~m} / \mathrm{s}^{2}$ with rotational velocities of about 1750 rounds per second. Finally, translational triaxial $(X-Y-Z)$ accelerometers embedded in the shell with their recording supplies and devices were used. These accelerations were measured in large caliber (for example, $120 \mathrm{~mm}$ ) artillery shell [20]. The experimental difficulty is that the axial (X) accelerations is considerably higher than the transverse values (of about 5 times lesser [21]). Then, if the $X$ measurement channel is not perfectly aligned with the shell trajectory, a part of the axial quantity can be transferred to the $Y-Z$ accelerometers and will mask the transverse quantities.

Concerning the data acquisition there are three existing methods: (i) recording by data-loggers embedded in the shell [22]; (ii) real-time recording by wire data transmission (the wire is stretched from projectile nose to an aligned point outside the tube); (iii) real time recording by optical transmission. Method (i) has been classically used both in large $(120 \mathrm{~mm})$ and medium caliber $(40 \mathrm{~mm})$ shell. For example, [9] record the strain signals from gages welded on small APFSDS projectile during the IB phase and correlated them with FEM simulation results. Method (iii) requires the coding of the acceleration measurements voltage through an optoelectronics system. The disadvantages of method (i) is that limited space is available for recording devices and supply in $25 \mathrm{~mm}$ shells, therefore limiting the amount of recorded data. Method (ii) cannot be used due to the rotational movement of the shell imposed by the inner tube engraving. Method (iii) based on $X-Y-Z$ accelerometers is potentially a good candidate for the aim of the present work: it has been chosen and will be described in Section 4 .

The experimental approach was guided by FEM simulations, which helped to forecast the order of magnitude of the accelerations, velocities, displacements, and strains. The experimental method is based on direct measurements during the IB and telemetry transmission of the data. In 1984, a presentation made by [23] explained the interest of this approach. However, it was not yet operated at that time.

The FEM model presented in this article relies to a large extent on the methodology developed by [24] and [11] for assessing the life time of medium gun barrels. Wear and thermo-mechanical coupling are not considered in the study. It was assumed that the barrel conditions are representative of the average functioning conditions found in practice.

In the first section the "ideal" or prescribed kinematic of the projectile is recalled. In the second section, the experimental methods are presented. In the third section, the FEM model is presented. Lastly, in the fourth section, we propose a discussion of the results and present the limitation of the study.

\section{Projectile "ideal" and prescribed kinematics}

In what follows the absolute fixed frame of reference, of orthogonal axes $X-Y-Z$, respectively with unit vectors $\mathbf{i}, \mathbf{j}$, $\mathbf{k}$, has its origin $\mathrm{O}$ at the tube entrance ( $X$ lies on the tube longitudinal axis, oriented towards the muzzle), see Fig. 1. 

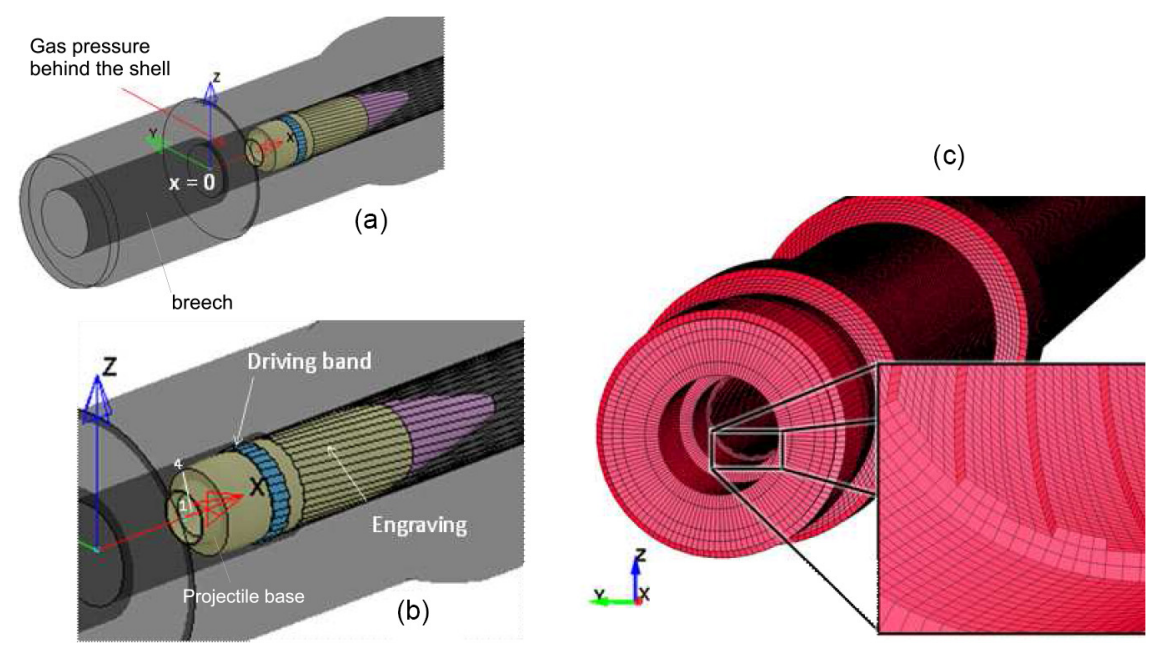

Fig. 1. Views of the projectile in the tube and of the engraving (a,b), which will guide the projectile rotation during its translational movement. The fixed reference frame orientation is shown in (a). (c) Provides a enlarged view of the mesh at the beginning of the tube.

In general, IB codes use the assumption that the longitudinal kinematic data of the projectile [acceleration $a_{x}(t)$ or velocity $v_{x}(t)$, or displacement $\left.x_{p}(t)\right]$ can be prescribed (imposed) deterministically by given time functions. These time functions are obtained separately by other specialized code processing the reactive flow and gas dynamics coupled to the projectile dynamics, but not to the tube dynamics response. This task is performed by ammunition suppliers for a given propulsion charge/ projectile couple. For our study, calculations of the longitudinal kinematic data are performed with the code "SIBIL" (which stands for: "Simulation de la Balistique Intérieure" in French) which provides for the user the aforementioned time functions. These functions are used as needed in the analytical processing. However, only the $x_{p}(t)$ tabulated values are required in the FEM model (Sect. 5 ) to prescribe the axial abscissa of the projectile.

The rifling engraved on the inner tube wall (Fig. 1) imposes a rotational movement to the shell, with angular velocity $\omega_{x}(t) \mathbf{i}$. The rifling is defined analytically by the thread $\beta(x)^{3}$, which is the axial distance traveled by the shell for one rotation (360 degrees). The time function $a_{x}(t)$ considered in this study is plotted in Figure 2b. From the rifling pitch $\beta(x)$, the instantaneous angular velocity $\omega_{x}(t)$

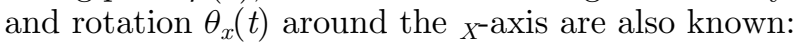

$$
\omega_{x}(t)=2 \pi \frac{v_{x}(t)}{\beta\left(x_{p}\right)}
$$

and

$$
\theta_{x}(t)=\int_{0}^{t} \omega_{x}(t) d t
$$

where $\beta(x)$ is evaluated according to the projectile abscissa $x_{p}(t)$.

\footnotetext{
${ }^{2}$ A Nexter-Munition company-designed IB code.

3 The function $\beta(x)$ is confidential
}
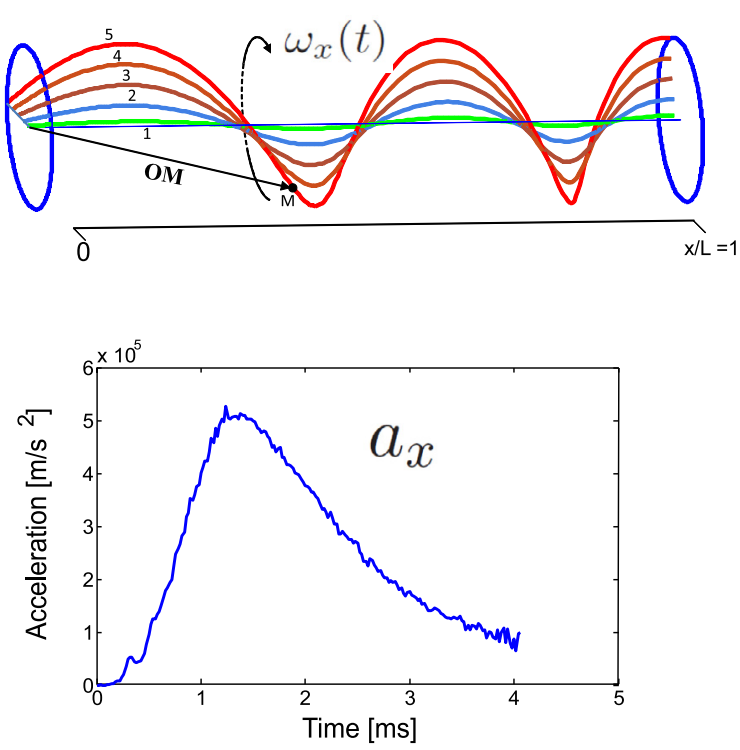

Fig. 2. Trajectories of five example points of the projectile (a) and longitudinal acceleration $a_{x}$ curve (b).

The trajectories of several points " $\imath$ " (1-5) of the projectile are plotted in Figure 2a. At time $t$, the "ideal" coordinates (along $X, Y, Z$ axes) of point $M_{i}$ having the radial distance $R_{i}$ are calculated as follow:

$$
\begin{aligned}
& X_{0}=x_{p}(t) \text { (prescribed) } \\
& Y_{0}=R_{i} \cos \left[\theta_{x}(t)\right] \\
& Z_{0}=R_{i} \cos \left[\theta_{x}(t)\right]
\end{aligned}
$$

For a given time velocity function, $v_{x}(t)$, the local curvature is specific to each moving point. Therefore, variation of the acceleration $\mathbf{a}_{\mathbf{N}}$ along the (instantaneous) normal $\mathbf{N}$ of the point trajectory will be influenced by local slopes and curvatures. In addition, due to straightness defects the real tube geometry does not match the ideal one provided by 
the Computer Aided Design plans. These local straightness variations also induce variations of vector $\mathbf{N}$ which, in turn, induce variations of the $\mathbf{a}_{\mathbf{N}}$ vector.

\section{Geometric effects}

For each point of the projectile, (for example, those represented in Fig. 2a), the maximum transverse acceleration is the acceleration $a_{N}=\left\|\mathbf{a}_{\mathbf{N}}\right\|$ along the normal vector $\mathbf{N}$ of its own trajectory. Consider any point $\mathrm{M}$ of the projectile (2a), with vector $\mathbf{r}=\mathbf{O M}$, and local curvature:

$$
\Gamma=\frac{1}{\rho}=\frac{1}{\|\mathbf{r}\| \mathbf{3}}\|\mathbf{r} \wedge \dot{\mathbf{r}}\|
$$

the tangential unit vector is $\mathbf{T}=\mathbf{r} /\|\mathbf{r}\|$. From vector $\mathbf{T}$ we obtain vector $\mathbf{N}$ according to:

$$
\mathbf{N}=\frac{1}{\dot{s}} \Gamma \mathbf{T}
$$

where: $s$ is the curvilinear abscissa and $\dot{s}=v$, the velocity vector modulus.

Lastly, the acceleration along the direction of $\mathbf{N}$ is given by:

$$
\left\|\mathbf{a}_{\mathbf{N}}\right\|=\left|\frac{v^{2}}{\rho}\right|
$$

When the projectile moves in a flexible tube, coordinates $X$, $Y, Z$ of point $\mathrm{M}$ are composed of a dynamic part $x, y, z$, and a static part $x_{0}, y_{0}, z_{0}$, representing the initial tube shape, so that:

$$
\begin{aligned}
& X=x_{p}(t) \text { (prescribed) } \\
& Y=y+y_{0} \\
& Z=z+z_{0}
\end{aligned}
$$

Coordinates, $y_{0}, z_{0}$, are only functions of the longitudinal abscissa $x_{0}$. Coordinates $y$ and $z$, or $Y$ and $Z$, are obtained from the numerical finite element model (see Sect. 5). The projectile trajectory $(X, Y, Z)$ in the tube is plotted in Figure 3.

- If the "ideal" kinematics along a perfectly straight line is considered, the $Y$ and $Z$ coordinates are respectively replaced by $Y_{0}$ and $Z_{0}$ equation 8 :

$$
\begin{aligned}
& X=x_{p}(t) \text { (prescribed) } \\
& Y=Y_{0} \\
& Z=Z_{0}
\end{aligned}
$$

- If the "ideal" kinematics along a non-perfectly straight line is considered, the $Y$ and $Z$ coordinates are respectively replaced by: $Y_{0}+y_{0}(x)$ and $Z_{0}+z_{0}(x)$ :

$$
\begin{aligned}
& X=x_{p}(t)(\text { prescribed }) \\
& Y=Y_{0}+y_{0}(x) \\
& Z=Z_{0}+z_{0}(x)
\end{aligned}
$$

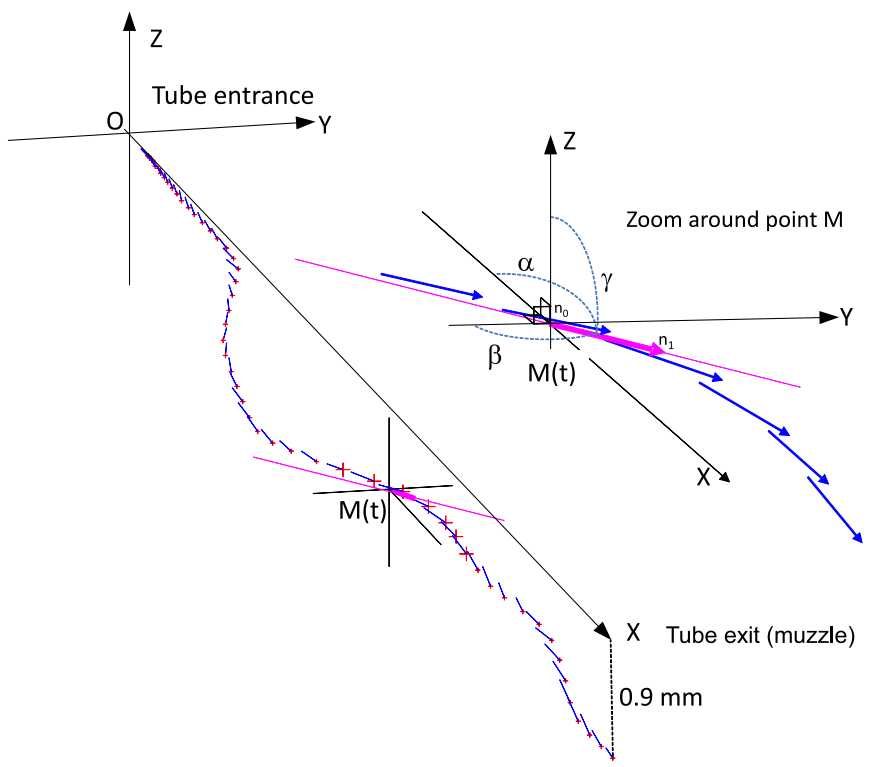

Fig. 3. $X-Y-Z$ projectile trajectory, (Eq. (8)). Each vector (arrow) represents the axis of the projectile at its instantaneous location.

As the local slope and curvature discovered by the projectile depend on the prescribed $x_{p}(t)$ and $v_{p}(t)=\dot{x}_{p}(t)$ time functions, the first and second derivatives of the static part enter the Cartesian components of the velocity and acceleration vectors respectively as:

$$
\begin{aligned}
& v_{x} \quad(\text { prescribed }) \\
& v_{y}=\frac{\mathrm{d} y}{\mathrm{~d} t}+v_{x} \frac{d y_{0}}{d x_{0}} \\
& v_{z}=\frac{\mathrm{d} y}{\mathrm{~d} t}+v_{x} \frac{d z_{0}}{d x_{0}} \\
& a_{x} \quad \text { (prescribed) }
\end{aligned}
$$

$$
\begin{aligned}
a_{y} & =\frac{d^{2} y}{d t^{2}}+2 v_{x} \frac{\partial^{2} y_{0}}{\partial t \partial x_{0}}+v_{x}^{2} \frac{d^{2} y_{0}}{d x_{0}^{2}}+a_{x} \frac{d y_{0}}{d x_{0}} \\
& =\frac{d^{2} y}{d t^{2}}+v_{x}^{2} \frac{d^{2} y_{0}}{d x_{0}^{2}}+a_{x} \frac{d y_{0}}{d x_{0}} \\
a_{z} & =\frac{d^{2} z}{d t^{2}}+2 v_{x} \frac{\partial^{2} z_{0}}{\partial t \partial x_{0}}+v_{x}^{2} \frac{d^{2} z_{0}}{d x_{0}^{2}}+a_{x} \frac{d z_{0}}{d x_{0}} \\
& =\frac{d^{2} z}{d t^{2}}+v_{x}^{2} \frac{d^{2} z_{0}}{d x_{0}^{2}}+a_{x} \frac{d z_{0}}{d x_{0}}
\end{aligned}
$$

For a given time velocity function, $v_{x}(t)$, the acceleration $\mathbf{a}_{\mathbf{N}}$ depends on the local curvature which may be specific to each moving point $\mathrm{M}$. One has to remind oneself that the real geometry is far from the ideal one plotted in Figure $2 \mathrm{a}$ due to straightness defects which modify the perfect helical path. This will be considered in the discussion, Section 6 . 


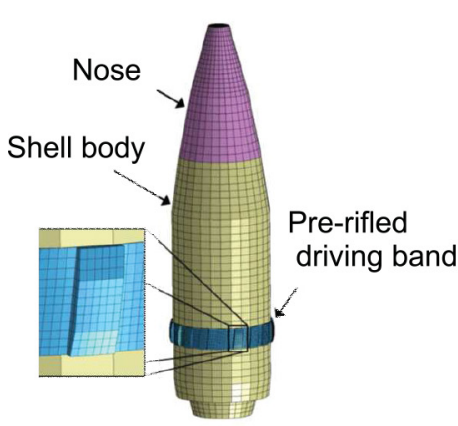

(a)

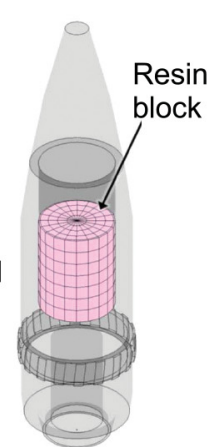

(b)

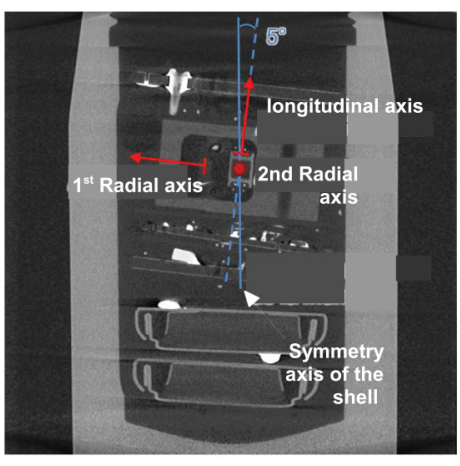

(c)

Fig. 4. (a) FEM of the projectile, (b) inside of the FEM projectile showing the resin block, (c) X-ray image of the real projectile showing the measurement device and their supplies. The mesh lines have been removed for clarity.

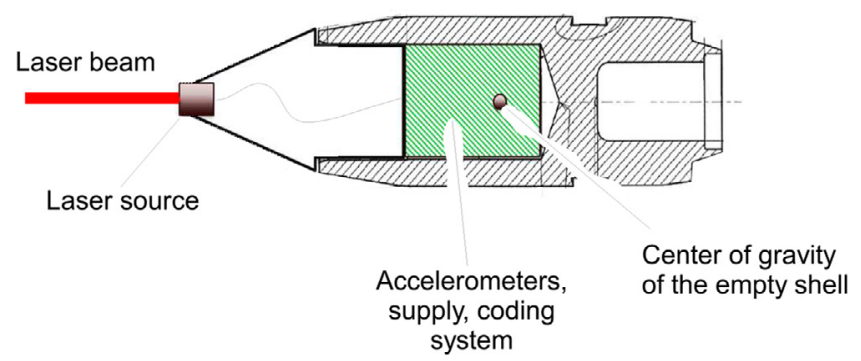

Fig. 5. Schematic of the instrumented projectile.

\section{Real acceleration measurements during the gun firing tests}

A challenging problem was to measure directly the transverse and axial accelerations during the IB phase and to transmit the data outside the tube. Specialized medium caliber shells were equipped with miniaturized accelerometers (manufactured by PCB) and their transducers connected to an opto-electronical transmission device. The whole measurement system designed and assembled by ATCOM Company was embedded inside a resin block (Fig. 4c) placed within the shell body and was designed to resist to the ballistic propulsive acceleration (about several times $10^{5} \mathrm{~m} / \mathrm{s}^{2}$ ) of the ammunition. First, the analogic voltages from the accelerometers are encoded in binary sequences which are translated to corresponding sequences of "off" and "on" state of emission of a LASER light beam through a lens mounted at the projectile apex, Figure 5. The lens enables the focalization of the light towards the gun muzzle. The light beam is then deviated by an oblique mirror (placed in front of the muzzle, Fig. 6) towards the Fresnel lens of an opto-receiver connected to the acquisition system. Then the received signal is decoded so that the binary acceleration data may be converted to physical values. The system provides four measurement channels (Ch1 to 4): CH1, is for the axial acceleration, $A_{X}$, $\mathrm{CH} 2$, and $\mathrm{CH} 3$ are for the transverse accelerations (denoted

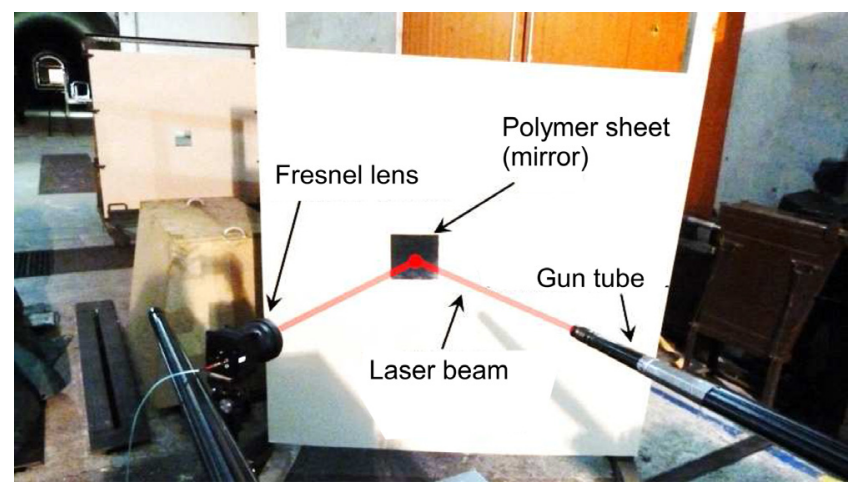

Fig. 6. Photograph of the experimental set-up used for the firing tests.

by $A_{i}$ where $i$ is the channel number) and $\mathrm{CH} 4$ is a control channel, which emits a continuous signal enabling to detect any interruption of the transmission.

For each test, circumferential strains were measured at various locations on the external tube wall. At the tube muzzle (exit section), several points displacements were measured by inductive sensors in the horizontal $(Y)$ and vertical $(Z)$ planes.

\section{The finite element model}

To assess the tridimensional dynamics of the system, simulations based on a finite element model elaborated with the LS-Dyna software were conducted. The software provides explicit hydrodynamics tools able to manage short and intense transient mechanical events as well as highly dynamic phenomena with possible non-linearity due to contact, geometry, or material in-homogeneities. Explicit calculations are made, in order to capture the fast dynamic events of the barrel projectile coupling. Total number of elements is 623646 .

The material of the tube is steel with a high elastic limit. The tube is modeled by an isotropic elasto-plasticity model. The material properties are defined by tensile tests on testsamples taken from the tube. 
To prevent non-convergence and aberrations of calculations, the barrel and projectile are meshed with hexahedrons elements. A view of the model is presented in Figure 1.

The barrel translational degrees of freedom are blocked in the three directions at the projectile entrance (these conditions represent the clamped boundary at the interface with the other part of the weapon system).

The projectile axial position is prescribed by the $x_{p}(t)$ time function obtained separately with the SIBIL code.

The contact logic chosen for the finite element model is the contact called CONTACT_AUTOMATIC_SURFACE_TO_SURFACE, a classical penalty method. This type of contact is recommended if important strains arise, for example in the crash simulations. In the present case, it has been chosen because high strains appear at the projectile-tube contact area (band interface, Fig. 4) as it was clearly demonstrated by [11]. In brief, the external diameter of the projectile is slightly larger than the internal tube diameter in order to have a perfect sealing of the gas (as a consequence, the projectile passage gives rise to important and rapidly growing circumferential strains). The contact is defined by the static coefficient of friction $f_{s}$ and the dynamic coefficient of friction, $f_{d}$. These parameters permit to correlate the model with the experimental data. Some studies ([19]) use $f_{s}=0.1$ and $f_{d}=0.13$.

The gas pressure acting on the barrel wall (behind the projectile base, Fig. 1) is programmed using the technique of progressive loading [11], which consist of dividing the tube in 60 virtual sections. In each section a time pressure function is defined so that the pressure climbs to its loading values when the projectile uncovers the element row of the section. This loading has been discussed in [11]. Between the breech and the instantaneous projectile base, the pressure verifies the linear gradient distribution.

The pressure of the propulsive gas is given by preliminary IB calculations which compute the gas dynamics associated with a given ammunition type. This approach is generally chosen in gun dynamics. Setting up a fluid-structureinteraction simulation, including the propellant gases is too complicated for the purpose of the study. Instead a weak coupling was applied. The base pressure is applied to the rear section of the projectile. The maximum base pressure is about some hundreds of MPa. At a given time, the pressure distribution between the breech and the projectile verifies the linear gradient distribution. So, behind the projectile, the pressure evolves as a combination of the breech and base pressure, weighted by the projectile location. In each section, gas pressure change (time pressure function). The quantity of gas increase, at the same time as the free volume between the breech and the projectile increase too. Then the volume increases faster, which creates a decrease in pressure. As in previous studies [3], the increase of pressure is supposed to be linear. At a given location, the pressure applied on the inner wall starts at 0 , and then climbs rapidly to the instantaneous base pressure applied on the projectile at the passing time and at the location of the row of elements materializing the local cross-section. The times when the projectile uncovers a given row of elements of the barrel were pre-calculated [with the $x_{p}(t)$ function], and a specific pressure vs. time curve (load curve) was defined for this row of elements: this starts at 0 when the band passes the lower boundary of the row of elements and reaches the base pressure when the projectile passes the upper boundary of the row. Applying this progressiveness to a sufficiently large number of element rows, smoothing the rise in pressure and limits the numerical problems. The procedure chosen to describe the moving pressure front and its rise time has been checked using the beam analytical solution published by [25]. Instead of a pressure, a punctual force was driven over a finite element beam model in the numerical code. The length of the element rows, which were loaded step by step, was the same as in the barrel model. The results were very close to the reference solution.

At time zero, the projectile-tube system is at rest. However, the initial static state of the tube takes into account the gravity load and straightness defects. In one of the phases of the manufacturing process, called "mechanical straightening", the tube rests on two simple supports and is loaded by a punctual force ${ }^{4}$ in order to optimize its straightness. However, it remains a level of uncertainty about the real tridimensional shape of the tube. To overcome this uncertainty, we have chosen to simulate this particular phase of manufacturing by replicating the mechanical straightening. The numerical load used for this preliminary calculation has been adjusted until the numerical straightness matches the measured straightness (not presented in this article). As a consequence, the tube model possesses a tridimensional straightness variations as well as residual stresses in the wall. Secondly, in the vertical plane (Z), the gravity droop was added by a numerical quasi-static calculation using the implicit solver of LSDyna. Note that the simplest curvature would be that of the gravity droop (deflection $F_{g}$ at the tube free end is then of the order of $1 \mathrm{~mm}$ ). The static profiles in the horizontal and vertical planes are respectively denoted $y_{0}$ and $z_{0}$.

The dynamic calculation by the explicit solver is then run, lasting about 75 hours to complete the simulation.

Projectile model. The projectile is meshed in four distinct parts (Fig. 4a), but with same element type (eight nodes hexahedrons): (i) the driving band, (ii) the shell body, (iii) the blunt nose, respectively with 12 100, 5500, 7500 elements. Materials are: sintered iron (elasto-plastic behavior) for the band, perfectly rigid for the shell body, aluminium (elastic) for the nose. The driving band and the shell body are linked by a tied contact. Within the shell body, the fourth part is an elastic resin block (Fig. 4b), modeled with 600 elements to represent the block where the measurement system is embedded (Fig. 4c). The driving band mesh is "pre-rifled", because the engraving process (when the shell enters the tube) is not modelled.

\section{Results and discussion}

\subsection{Longitudinal acceleration}

In Figure 7a, the axial acceleration $A_{X}$ obtained from the experiments is compared to the accelerations calculated by the IB code SIBIL. It is found that the two curves match very well. The discrepancy between the peak values is due

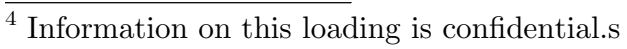



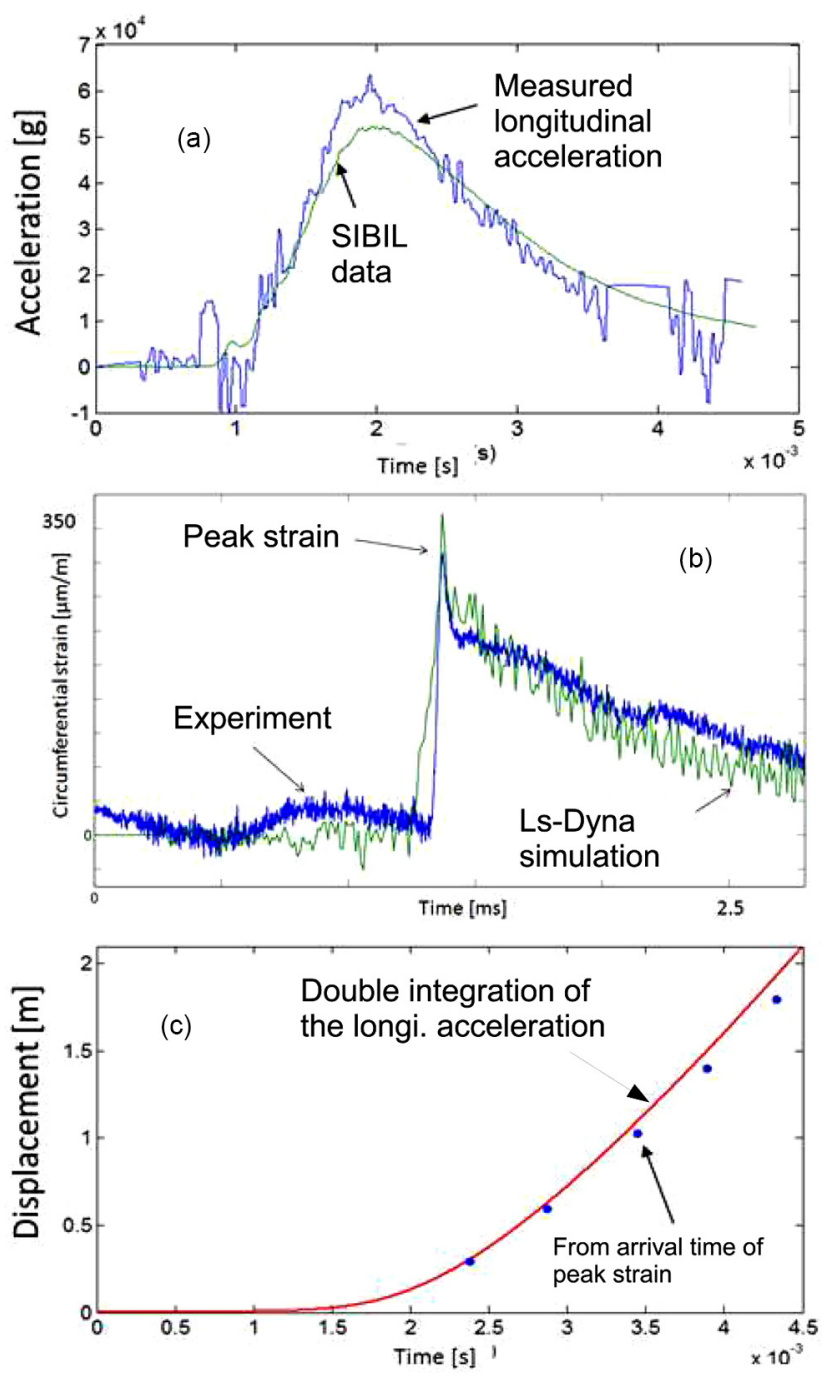

Fig. 7. Longitudinal measurements. (a) Comparison of the axial measured acceleration with pre-calculated "SIBIL" acceleration. (b) Comparison of the measured circumferential strains with the calculated quantities. (c) Axial projectile abscissa obtained by integrating twice the measured accelerations. Comparisons with the projectile detection by the peak strain signal.

to the difference between the architecture of the test ammunition and that processed by SIBIL. The test ammunition included the embedded measurement system and was a little lighter than the real one (with same charge). Now, as it is known that the projectile passage creates a peak value [11] in the circumferential strains (Fig. 7b), these strain histories were used to identify the arrival time of the projectile at the gage location, $X_{j}$. In Figure $7 \mathrm{c}$, the plots of $X_{j}$ vs. time is clearly well superposed with the projectile position obtained after two time integration of the experimental $A_{X}$ acceleration (filtered) history.

Then, it can be concluded that the axial acceleration is correctly applied in the simulation code, leading to expected axial kinematic data.

It is particularly satisfying to verify that the experimental acceleration provided by channel CH1 ( $x$-acceleration) replicate the SIBIL acceleration known in advance.
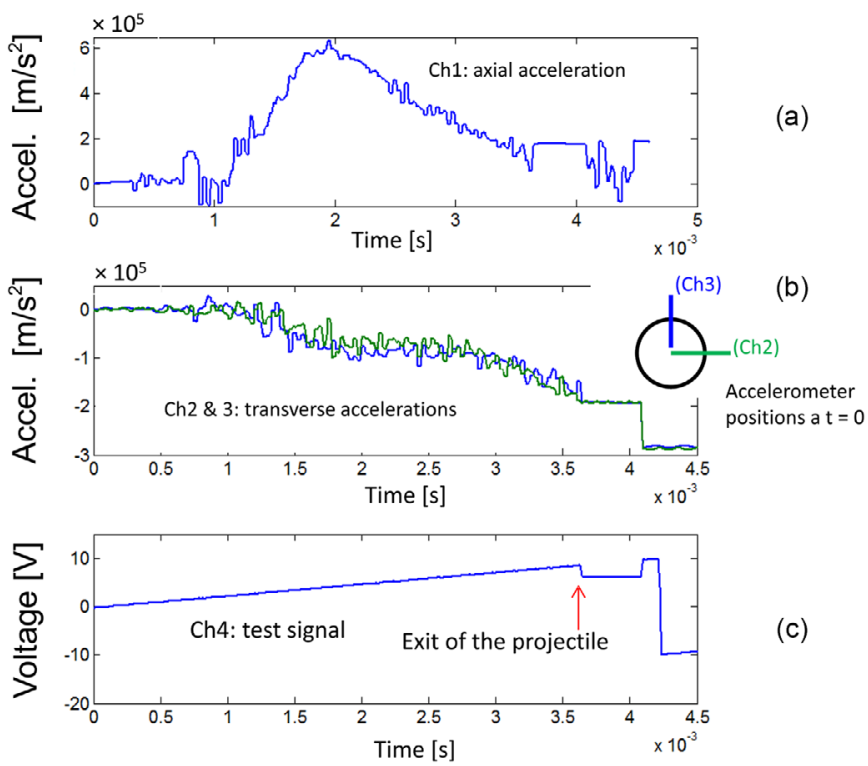

(c)

Fig. 8. Measurements of firing \# 6 showing the axial acceleration (a) transverse accelerations (b) and the control signal (c). In (b), positions (Ch2) and (Ch3) are those of the accelerometers and before ignition.

Moreover, the arrival times of the projectile detected by the circumferential peak strains measured at distinct gage stations are in accordance with double time integration of the experimental longitudinal acceleration curve (Fig. 5c) and with the pre-calculated $x_{p}(t)$ function. This is an argument in favour for prescribing pre-determined functions (here, $x_{p}(t)$ in the FEM model) in gun dynamics simulations, rather than computing a complete fluidstructure interaction of the gas dynamics coupled with the structural (shell and tube) dynamics.

\subsection{Analysis of the transverse acceleration measurements}

A typical example of raw measurement results is presented in Figure 8. In Figure 8c, Chanel 4 clearly indicates that not any parts of the signal is missing in the records since it is continuously displaying from the beginning until projectile exit (after $3.5 \mathrm{~ms}$ ). The consistency of the longitudinal physical acceleration was already confirmed by comparing results of Ch1 with the time curve provided by SIBIL (Fig. 7b).

In the experiments, the initial angular positioning of the accelerometers is determined thanks to grooves machined on the breech entrance. The scheme of Figure $8 \mathrm{~b}$ recalls that $\mathrm{Ch} 2$ and $\mathrm{Ch} 3$ are aligned with the $Z$ vertical and $Y$ horizontal axis respectively.

To analyze the results, the "ideal" normal acceleration calculated with the SIBIL axial acceleration $a_{x}(t)$ combined to the rotation $\omega_{x}(t)$ prescribed by the variable rifling pitch $\beta$ is compared to the measurements in Figure 9a. Then, the projectile trajectory is identical to the helical path of variable thread $\theta_{x}$, equation (8).

It is clearly seen that the real data does not match the "ideal" kinematic. 

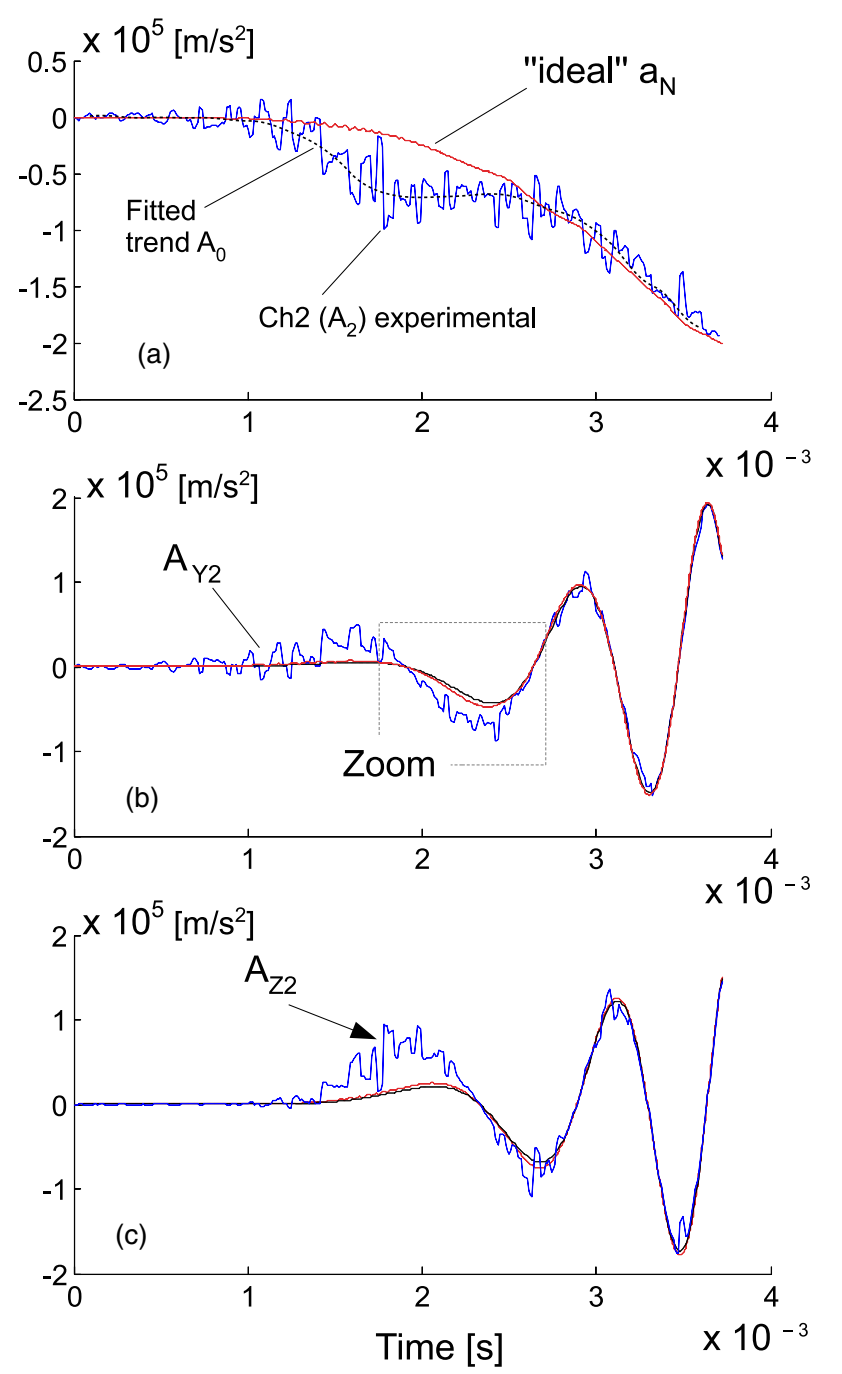

Fig. 9. (a) The acceleration on one transverse measurement direction (see Fig. 1) and on the fixed $Y$ and $Z$ fixed directions of the laboratory frame.

First, the fitted trend, $A_{0}$, reveals a low frequency oscillatory behavior of the projectile. To better highlight the differences between the measurements and the ideal results, we suppose that the angular accelerometer position in the $Y-Z$ plane is given by the angle $\theta_{x}(t)$ as calculated by equation (2). Then assumed experimental components are evaluated with:

$$
\begin{aligned}
& A_{Y i}=A_{i} \cos \left[\theta_{x}(t)\right] \\
& A_{Z i}=A_{i} \sin \left[\theta_{x}(t)\right]
\end{aligned}
$$

and plotted in Figure 9b and 9c. The deviations of the measurements with these Cartesian projections (indicated by arrows in Fig. 9b,c) are important in the beginning of the signal.

Secondly, oscillations of higher frequencies (small durations) are visible throughout the experimental signal. The question is to determine whether or not these high frequencies movements follow closely the tube vibrations

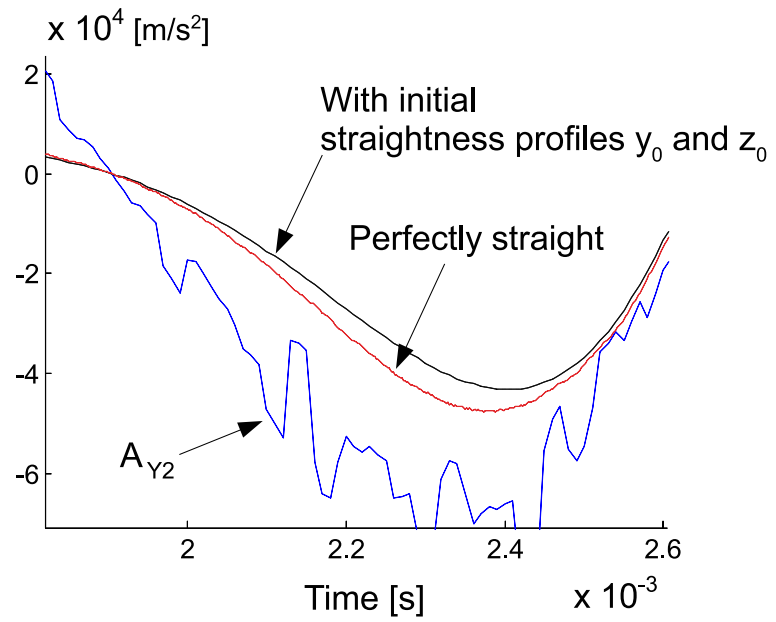

Fig. 10. Zoom on the $A_{Y 2}$ signal displayed in Fig. 9b.

or are own oscillations of the projectile, which means that the accelerometer axis undergoes small angular oscillations while the projectile does not completely follow the tube displacement.

Effects of the initial static profile $\left(x_{0}, y_{0}, z_{0}\right)$ can be highlighted if the first and second space derivatives (slope and curvature, respectively) of the static profile are included in equations (11) and (12). The $y_{0}$ and $z_{0}$ functions are given in equations (15) and (16) where parameters $\left(A_{h 1}, \mu_{h}, A_{h 2}\right.$, $\left.\mu_{v}, s, s_{1}, s_{2}\right)$, are deduced from geometrical measurements. Figure 11 shows the plots of these functions and of their second derivatives vs. the $X$ coordinate. The circle and star symbols indicate experimental data.

$$
\begin{gathered}
y_{0}(x)=\frac{A_{h 1}}{s_{1} \sqrt{2 \pi}} \mathrm{e}^{\frac{\left(x_{0}-\mu_{h}\right)^{2}}{2 s_{1}^{2}}}-\frac{A_{h 2}}{s_{2} \sqrt{2 \pi}} \exp \frac{\left(x_{0}-\mu_{h}\right)^{2}}{2 s_{2}^{2}} \\
z_{0}(x)=\frac{A_{v}}{s_{1} \sqrt{2 \pi}} \exp \frac{\left(x_{0}-\mu_{v}\right)^{2}}{2 s_{1}^{2}}-F_{g}\left(\frac{x_{0}}{L}\right)^{2}
\end{gathered}
$$

Indeed, slope and curvature have the largest influences where the velocity $v_{x}$ and acceleration $a_{x}$ take their lager values. The acceleration reaches its peak value at $2 \mathrm{~ms}$ when the projectile has traveled less than $50 \mathrm{~cm}$ (see Fig. 7a,c). The velocity is maximum near the tube muzzle.

The results of the calculation including the static profiles are plotted in dotted line in Figure 9b,c, and in Figure 10 (which is a zoom made in Fig. 9b). From these results it appears that functions, $y_{0}, z_{0}$, and their derivatives, have little influences on the results. This is due to the small values of the first and second derivatives: the latter reached the maxima in less than $0.01 \mathrm{~m}^{-1}$.

Figure 12 presents the FEM model result. This model failed to faithfully predict the essential feature of the observed signal: the low frequency $A_{0}$ and the higher frequency oscillations. However, this model is moderately satisfactory since it predicts roughly the transverse acceleration $A_{i}$ but very accurately the circumferential stresses in the tube wall. It is believed that increasing the mesh refinement and optimizing both the boundary 

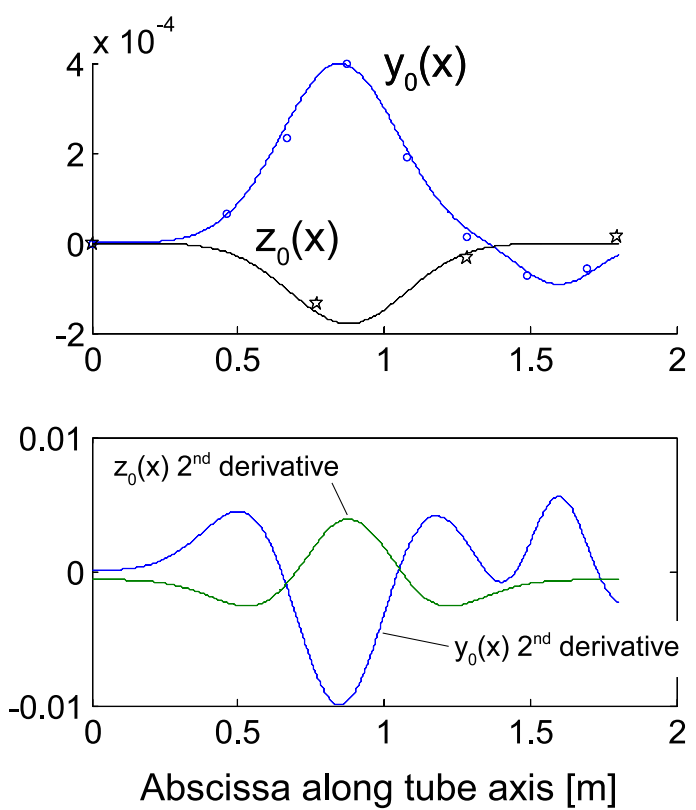

Fig. 11. Static profiles, $y_{0}(x), z_{0}(x)$, and their second space derivatives.

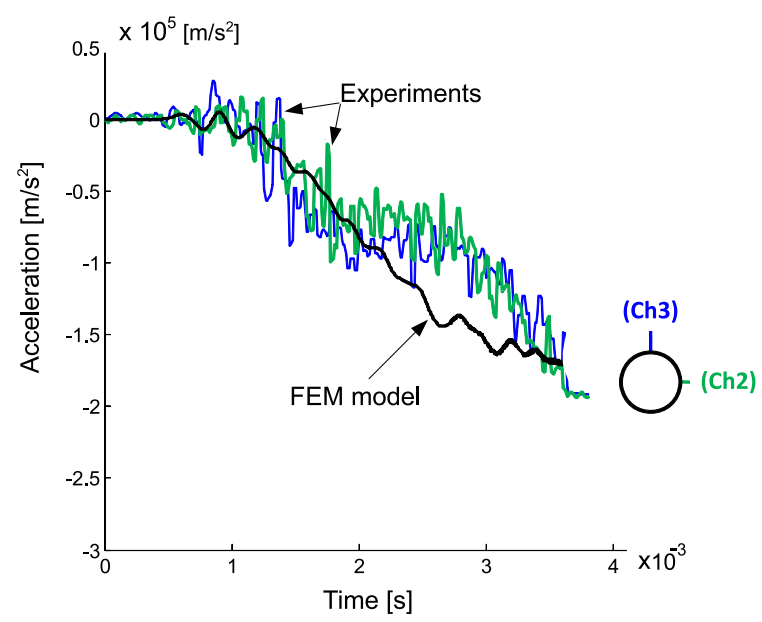

Fig. 12. Transverse numerical acceleration compared to the measured data.
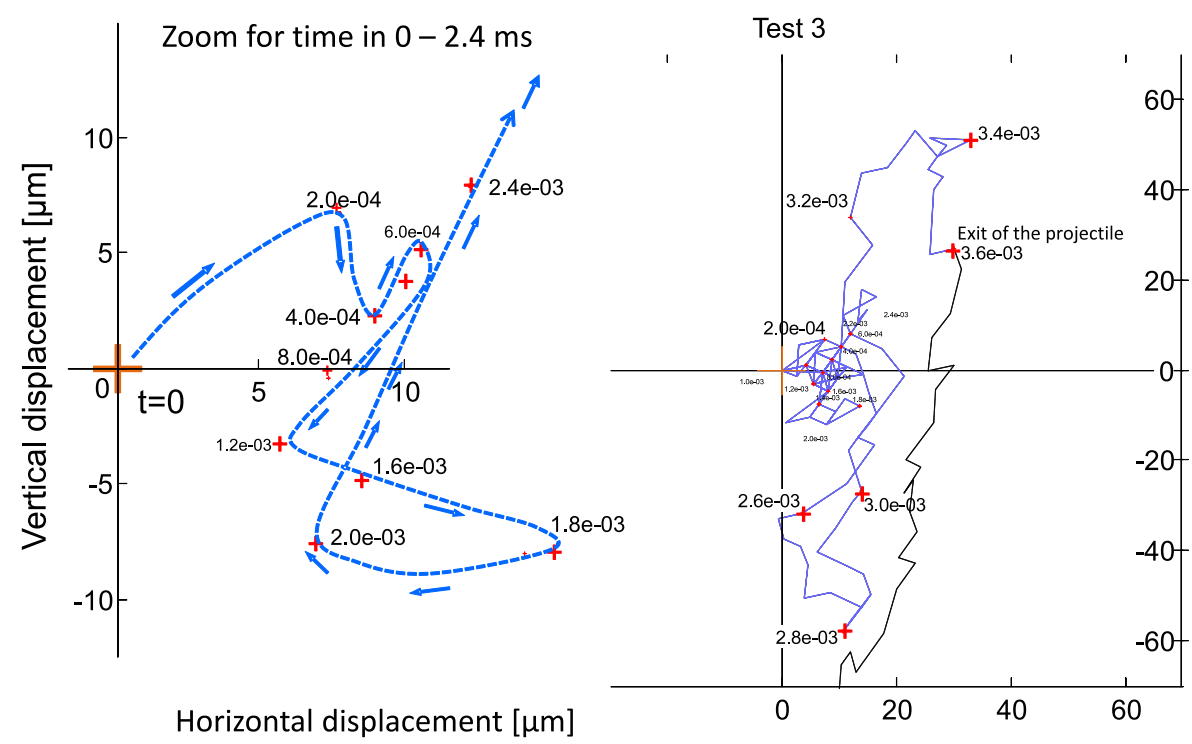

Fig. 13. Tube muzzle displacements measured in the $Y-Z$ plane.

conditions and the contact logic (tube-shell) will enable to obtain more realistic results. This goal is left for future study since the aim of the present work was to obtain direct acceleration measurements.

The mutual actions between the tube and the projectile consist of a torque as well as normal and tangential forces exerted through the contact area. This area is the projectile band. Resulting from these actions the displacement fields on the tube is 3D and space and time dependent. The complexity of the structural response is illustrated by Figure 13 showing vertical vs. horizontal measured displacements of a point located on the tube muzzle. Successive positions are ordered by their time values chosen in the displacement histories. After $3.5 \mathrm{~ms}$, the dashed line indicates that the projectile has exited the tube. This plot shows more obviously the accelerating rotation of the tube free end. In the $Y-Z$ plane, the curve drawn by the tube muzzle looks like a spiral. Starting from the center, the tube is deformed longitudinally (as shown by time and space variations of the longitudinal displacements, not presented here) and laterally; at the same time, the tube rotates along a non-fixed direction owing to the 3D stresses in the wall.

To conclude at this step, the low frequency carrier wave $A_{0}$ observed in the Ch2-Ch3 signals ( $A_{0}$ in Figs. $8 \mathrm{~b}$ and $9 \mathrm{a}$ ) is related to the combined rotational, bending and longitudinal acceleration. 
If the tube axis deviates significantly from the perfectly straight orientation, the product of the local slope and axial velocity, the product of the local curvature and the square axial acceleration, both contribute to the transverse acceleration variations. These contributions are combined to the contributions of the transverse accelerations transmitted by the tube at the instantaneous projectile location $\left(x_{p}\right)$. The latter are dynamics torsional waves, as well as bending and longitudinal waves.

\section{Conclusion}

The study succeeded in measuring the transverse accelerations during the in-bore travel of shells in a medium caliber gun. We have taken on the technical challenge of designing and operating a miniaturized system able to measure and transmit optically the data outside the tube during the firing. Owing to this difficulty, few results are available for this kind of weapon system. In a general way, the simulation model provides results that help to understand the experimental results (in particular the tube displacements). However, the numerical solution still lacks of accuracy regarding the high frequency components of the measurements. These may reflect more complex issues such as progressive wear of the band, geometrical inhomogeneities of the internal tube wall, which are likely to alter the regularity of the projectile contact area, leading to sporadic, sudden, (although of limited amplitudes), accelerations in the signal. This is what is observed in every signal provided by transversally mounted accelerometer in the shell.

Remaining work is then to improve the analysis of the data fluctuations, which could only be done with an improved physical-mechanical model and an optimized measuring system, in particular by optimizing the sensors positioning in the test ammunition and the positioning of projectile prior to firing.

\section{Notation}

$X-Y-Z \quad$ Axis of the orthonormal fixed frame (or global frame) having its origin at the tube entrance.

$x_{0}, y_{0}, z_{0} \quad$ Initial coordinates of the tube axis (static coordinates) in the global frame.

$x, y, z \quad$ Dynamic coordinates in the global frame.

$X_{0}, Y_{0}, Z_{0}$ "Ideal coordinates" on the helical rigid trajectory.

$X, Y, Z \quad$ (In italic) Coordinates of a point at time $t$ in the global frame.

$v_{x}, v_{y}, v_{z} \quad$ Theoretical velocity components in the global frame.

$v \quad$ Velocity vector modulus.

$a_{x}, a_{y}, a_{z} \quad$ Theoretical acceleration components in the global frame.

$A_{X} \quad$ Measured longitudinal acceleration.

\section{References}

[1] M. Chen, Projectile balloting attributable to gun tube curvature, Shock and Vibration, 17 (2010) 39-53
[2] M. Liennard, Dynamique des tubes parcourus à grande vitesse, Influence de la géométrie des tubes et leur environnement sur la justesse et la dispersion, $\mathrm{PhD}$ thesis, Université d'Orléans, France, 2015.

[3] D. A. Rabern and M. W. Lewis. Two end three-dimensional simulations of moving pressure fronts in gun tubes. Journal of Pressure Vessel Technology, 114 (1992), 181-188

[4] S.H. Chu, New approach for analysis of transverse projectiletube interactions, Proceedings of the 3rd US Army Symposium on Gun Dynamics, 1981.

[5] T. Simkins, Resonance of flexural waves in gun tubes, Technical Report ARCCB-TR-87008, US Army Armament Research, Development and Engineering Center, Watervliet, NY, 1987.

[6] Q. Lambert, A. Langlet, J. Renard, N. Eches, Dynamique en flexion de tubes parcourus à grandes vitesses. Mécanique \& Industries, 9 (2008) 559-569

[7] Y.A. Su, I.G. Tadjbakhsh, Transient vibrations and instability in flexible guns-I. Formulations, The International Journal of Impact Engineering, 11 (1991) 159-171

[8] Y.A.Su, I.G. Tadjbakhsh, Transient vibrations and instability in flexible guns- II. Response characteristics, The International Journal of Impact Engineering, 11 (1991) 173-184

[9] N. Eches, D. Cosson, Q. Lambert, A. Langlet, J. Renard, Modelling of the dynamics of a $40 \mathrm{~mm}$ gun during firing. 7th LS-DYNA Conference, http://www.dynalook.com/europe an-conf-2009/J-I-05.pdf, 2009.

[10] M.T. Soifer, Projectile motion in a flexible gun tube, http:// www.dtic.mil/dtic/tr/fulltext/u2/a149022.pdf, 1984.

[11] O. Chevalier, A. Langlet, L. Fouché-Sanseigne, Y. Guilmard, Assessment of the lifetime of gun barrels under high-speed moving loads, Journal of Pressure Vessel Technology, 137 (2014) 9 p.

[12] S. Deng, H.K. Sun, C.-J. Chiu, K.-C. Chen, Transient finite element for in-bore analysis of $9 \mathrm{~mm}$ pistols, Applied Mathematical Modelling, 38 (2014) 2673-2688

[13] D.N. Bulman, S.F. Storey. EM gun, an investigation into the dynamics during the launch process using the SIMBAD gun dynamics program, Report No. Simatics/EMGUN/0696, Simatics Ltd. June, 1996, UK Restricted - Commercial, 1996

[14] D. Eckenfels, S. Nezirevic, M. Schneider, Mesure de la vitesse d'un projectile dans le lanceur Â rails "EMA3" avec un radar Doppler. ISL, 2002.

[15] J. Bornstein, I. Celmins, P. Plostins, E.M. Schmidt. Techniques for the measurement of tank cannon jump, Ballistic Research Laboratory, Aberdeen Proving Ground, Maryland, 1988

[16] B.T. Haug, Optical device for determining small angular motion, Ballistic Research Laboratories, Aberdeen Proving Ground, Maryland, 1977.

[17] J.S. Owens, Modeling and simulation: gun launch dynamics and aeroballistic analysis via onboard laser diode, ATK Advanced Weapons/WV, 2011.

[18] M.J. Wilson, Attitude determination with magnetometers for gun-launched munitions, Weapons and Materials Research Directorate, ARL, Aberdeen Proving Ground, 2004.

[19] M.T. Soifer, R.S. Becker, Projectile motion in a flexible gun tube. ADA149022, https://archive.org/details/DTIC_ADA149022, 1984.

[20] D.M. Grzybowsky, P.J. Peregino, B.S. Davis, Development of a telemetry-enabled high-G projectile carrier. Weapons and Materials Research Directorate, ARL, 2012.

[21] J. Zu, T. Ma, D. Pei, J. Fan, H. Du, New concept dynamic test on ballistic parameters measurement, 25th International Symposium On Ballistics, 2010. 
[22] V. Wegner, L. Kocher, Mesure des accélérations Ã bord des projectiles lors du départ du coup, Institut Saint-Louis (ISL), 1987.

[23] V. Wegner, Syst $\ddot{\widetilde{A}}$ me de télémesure pour la transmission de données Ã partir de projectiles pendant la phase d'accélération dans le tube du canon, Institut Saint Louis, conférence européenne sur la télémétrie, 1984.
[24] O. Safont, Méthodologie de dimensionnement de tubes en dynamiquePhD thesis, Université d'Orléans, France, 2011.

[25] A. Langlet, O. Safont, J. Renard, The response of infinite strings and beams to an initially applied moving force: analytical solution, ASME Journal of VibRation and Acoustics, 134 (2012)

Cite this article as: M. Liennard, O. Chevalier, A. Langlet, Y. Guilmard, M. Mansion, An analysis of the accelerations of a projectile in a gun tube by direct measurements and telemetry of the data, Mechanics \& Industry 19, 406 (2018) 